\title{
Lumbar artery pseudoaneurysm: a rare complication of a bone marrow aspiration and trephine biopsy
}

\author{
Sandstrom A* and Pankaj J \\ Department of Surgery, Nambour Hospital, Australia
}

\begin{abstract}
Introduction: Bone marrow aspiration and trephine biopsy (BMAT) is a procedure performed for the diagnosis and staging of haematological disorders. The usual site for a BMAT is the posterior or anterior iliac crest. Complications are uncommon and include soft issue trauma, haemorrhage and infection with haemorrhage being the most frequent and more likely to occur in patients who are thrombocytopenic or anticoagulated.

Case description: This is a case report of a patient who presented with right lower back pain and swelling at the site of a BMAT biopsy that was performed six weeks previously. Significant medical history included a newly diagnosed myelodysplastic disorder and the patient had a platelet count of $30 \times 10^{9} / \mathrm{L}$ on presentation. A computed tomography angiography revealed a lumbar artery pseudoaneurysm. It was treated with ultrasound guided compression with complete resolution.

Discussion: Gluteal and iliac artery pseudoaneurysms as a complication of BMAT have been reported in the literature. Themre are no other cases of a lumbar artery pseudoaneurysm occurring after a BMAT procedure. Treatment for lumbar artery pseudoaneurysm includes open surgical and endovascular options. In this case, ultrasound guided compression was performed with good effect.
\end{abstract}

\section{Introduction}

Bone marrow aspiration and trephine biopsy (BMAT) is an investigation for the evaluation of haematological conditions, cancer and metastatic disease as well as storage disorders. Relative contraindications to BMAT include severe disseminated intravascular coagulopathy or other severe bleeding disorders [1], local skin infections and ostemyelitis. The preferred site is the posterior or anterior iliac crest and other potential sites include sternum, tibia, femur, vertebral bodies and ribs. Complications are uncommon and estimated at 0.05 0.07 percent with the most frequent being haemorrhage particularly in patients who are thrombocytopenic or on anticoagulants [2]. Rarely, gluteal or internal iliac artery pseudoaneurysms have been reported [3]. This case study describes a lumbar artery pseudoaneurysm occurring secondary to a BMAT of right iliac crest. There have been no previous cases of a lumbar artery pseudoaneurysm related to BMAT reported in the English literature.

\section{Case}

A 60 year old female presented with increasing right lower back pain and swelling at the site of a BMAT that was performed six weeks prior. The results of this BMAT were consistent with myelodysplastic syndrome. Her past medical history included large cell lung carcinoma 8 years prior that was treated with left lobectomy and chemotherapy. On presentation her platelet count was $30 \times 109 / \mathrm{L}$ and her haemoglobin level was $92 \mathrm{~g} / \mathrm{L}$. An ultrasound demonstrated a hypoechoic cavity measuring $37 \times 20 \times 23 \mathrm{~mm}$ with internal vascular flow favouring a pseudoaneurysm. A computed tomography angiography (CTA) confirmed a pseudoaneurysm of a lumbar artery branch overlying the right iliac bone (Figures 1 and 2). It was planned to treat this with thrombin injection. In consultation with the haematology team, a unit of platelets was given which brought her platelet count to $50 \times$
109/L. During the procedure, the interventional radiologist found the pseudoaneurysm to be largely thrombosed and so it was treated with ultrasound guided compression alone with good immediate result. The patient was discharged and an ultrasound scan performed one week later showed a completely thrombosed pseudoaneurysm with no internal flow.

\section{Discussion}

The complications of BMAT are rare and include haemorrhage, soft issue trauma and infection. Isolated reports of retroperitoneal haemorrhage, fractures of underlying bone and gluteal or iliac artery pseudoaneurysms and gluteal compartment syndrome are found in the literature [1]. The usual site of bone marrow biopsy is the posterior superior iliac spine (PSIS) of the iliac crest. The two approaches used are the perpendicular approach advancing the needle anteriorly and the lateral approach in which the needle is directed towards the ipsilateral anterior superior iliac spine (ASIS). In a study of human cadavers by Konda et al, it was found that the lateral approach was less lively to cause neurovascular injury compared with the perpendicular approach [4]. In order to avoid complications of pseudoaneurysm and haemorrhage in the first instance, the recommendation is for compression of the biopsy site until adequate haemostasis is achieved followed by the application of compression bandage for twenty-four hours [3].

Correspondence to: Anna Sandstrom, Nambour Hospital, Hospital Road, Nambour 4560, Australia, Tel: (+617) 5470 6600; E-mail: anna.sandstrom@ health.qld.gov.au

Key words: bone marrow examination, lumbar artery, aneurysm, false, vascular surgical procedures, endovascular procedures

Received: July 10, 2017; Accepted: August 09, 2017; Published: August 12, 2017 


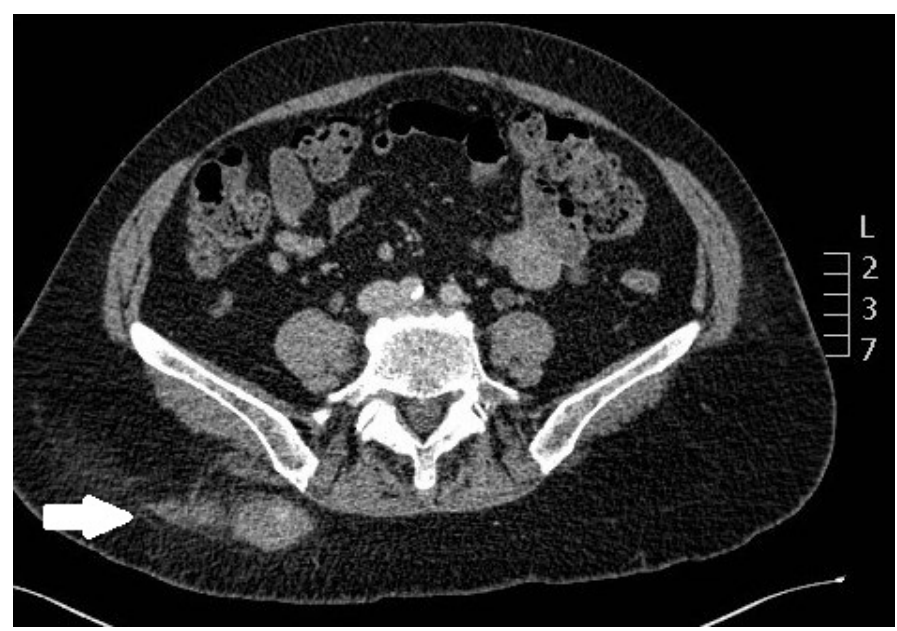

Figure 1. Computed tomography angiography: axial view of the lumbar artery pseudoaneurysm.

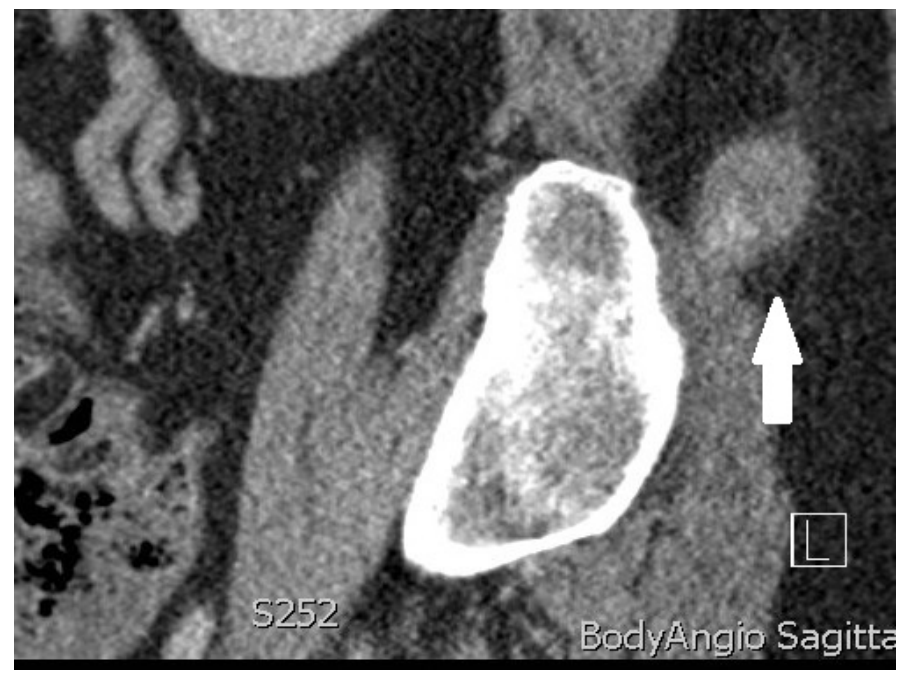

Figure 2. Computed tomography angiography: sagittal view of the lumbar artery pseudoaneurysm.

There are only a handful of cases of superior gluteal artery or internal iliac artery injury during a BMAT procedure reported in the literature and none pertaining to lumbar artery injury. Sullivan described a fifty-five year old patient who presented with superior gluteal artery pseudoaneurysm one hour after a BMAT procedure. It was complicated by gluteal compartment syndrome which was treated successfully with coil embolization [5]. Bailey reported a case of internal iliac artery aneurysm in an infant two days following a BMAT that again was managed endovascularly with coil embolization [3]. In a case report by Charisa, a twenty-nine year old male developed hypovolaemic shock one hour after a BMAT and was found to have massive retroperitoneal haemorrhage and a superior gluteal pseudoaneurysm on imaging. Endovascular coiling was attempted which unfortunately was unsuccessful. He developed abdominal compartment syndrome and due to significant congenital cardiac disease, no further surgical intervention was undertaken and he died two days later [6]. According to the literature, the recommended management of superior gluteal artery pseudoaneurysms includes direct pressure, angiographic embolisation and open surgical procedures [2-4].

There are no reports in the literature of lumbar artery pseudoaneurysms (LAPA) following BMAT. The lumbar arteries are small paired vessels that originate from the dorsal aspect of the abdominal aorta at the level of the transverse processes from L1 to L4. Pseudoaneurysms of the lumbar artery are rare and the etiology can be iatrogenic, traumatic, infective or spontaneous with the most frequent cause being penetrating abdominal trauma. Reports of iatrogenic injury relate to aortic repair, renal surgery, spinal anaesthesia and lumbar disc surgery. Patients with bleeding tendencies or connective tissue disorders are at an increased risk. Rupture of a lumbar pseudoaneurysm can present with acute flank pain, hypovolaemia, anaemia or retroperitoneal hematoma [7].

Lumbar artery pseudoaneurysms are best identified with computed tomography angiography (CTA). Treatment for LAPAs mainly includes open surgical and endovascular options. Endovascular embolization has been recommended in the literature as a safe and effective method to manage LAPAs in both the scenario of active extravasation as well as in prophylactic prevention of future rupture. A surgical incision is avoided and the pseudoaneurysm can be precisely identified [8]. In a study of trauma patients by Sofocleous et al, eleven patients with lumbar artery injuries received successful selective embolization using either coils, gelfoam or particles [9]. An alternative treatment option is percutaneous thrombin injection; however, this technique is dependent on anatomic ease. Indications for surgical intervention includes patients with refractory shock, failure of angiographic intervention or in patients where contrast medium is contraindicated.

\section{Conclusion}

To our knowledge, although several cases in the literature reported pseudoaneursysms of the superior gluteal and internal iliac artery, this is the only case in the literature of a lumbar artery pseudoaneurysm following a BMAT. It was managed with direct pressure alone which resulted in successful thrombosis of the pseudoaneurysm. LAPAs are a potentially life threatening pathology and should be considered in patients that present with flank pain and hemodynamic instability following a BMAT procedure.।

\section{References}

1. Malempati S, Joshi S, Lai S, Braner DA, Tegtmeyer K (2009) Videos in clinical medicine. Bone marrow aspiration and biopsy. N Engl J Med 361: e28.

2. Bain BJ (2003) Bone marrow biopsy morbidity and mortality. Br J Haematol 121 949-951. [Crossref]

3. Bailey MA, Farquharson NR, Coughlin PA, Vora A, Puppala S, et al. (2011) Internal iliac artery pseudoaneurysm in an infant following bone marrow trephine biopsy. Br J Haematol 153: 1. [Crossref]

4. Konda B, Pathak S, Edwin I (2014) Safe and successful bone marrow biopsy: an anatomical and CT-based cadaver study. Am J Hematol 89: 943-946. [Crossref]

5. Sullivan CM, Regi JM (2013) Pseudoaneurysm of the superior gluteal artery following bone marrow biopsy. Br J Haematol 161: 289-291. [Crossref]

6. Chamisa I (2007) Fatal vascular retroperitoneal injury following bone marrow biopsy S Afr Med J 97: 246. [Crossref]

7. Liu P, Chiu TF, Li CH (2012) Spontaneous lumbar artery pseudoaneurysm bleeding: a case report. J Emerg Med 42: 299-300.

8. Oh YM, Choi HY, Eun JP (2013) Delayed Retroperitoneal Hemorrhage due to Lumbar Artery Pseudoaneurysm after Lumbar Posterolateral Fusion. J Korean Neurosurg Soc 54: 344-346. [Crossref]

9. Sofocleous CT, Hinrichs CR, Hubbi B, Doddakashi S, Bahramipour P, et al. (2005) Embolization of isolated lumbar artery injuries in trauma patients. Cardiovasc Intervent Radiol 28:730-735. [Crossref]

Copyright: (O2017 Sandstrom A. This is an open-access article distributed under the terms of the Creative Commons Attribution License, which permits unrestricted use, distribution, and reproduction in any medium, provided the original author and source are credited. 